\title{
Long-standing Dermatitis Treated With Dupilumab With Subsequent Progression to Cutaneous T-cell Lymphoma
}

\author{
L. Claire Hollins, MD; Paul Wirth, MD; Gregory J. Fulchiero Jr, MD; Galen T. Foulke, MD
}

\section{PRACTICE POINTS}

- Dupilumab is a safe and effective treatment for atopic dermatitis (AD) in both children and adults.

- Prior to starting treatment for presumed adultonset $A D$, consider smoldering cutaneous T-cell lymphoma (CTCL).

- Dupilumab may interact with the cutaneous immune system, leading to an expedited presentation of CTCL in patients with chronic adult-onset $A D$.

This case series discusses 3 patients with long-standing eczematous or psoriasiform dermatitis, demonstrated by multiple biopsies. Following off-label treatment with dupilumab, all 3 patients had clinical expansion of disease, with histopathologic features consistent with cutaneous T-cell lymphoma (CTCL) on subsequent biopsy. We postulate that this expansion likely was secondary to an exacerbation of extant CTCL following exposure to dupilumab. A proposed mechanism of promotion of CTCL is based on the functional increase in $\mathrm{IL}-13$ available for binding at the upregulated IL-13 receptor (IL-13R) $\alpha 2$ site on cells, following blockade of the $\alpha 1$ receptor with dupilumab. This progression merits further investigation.

Cutis. 2020;106:E8-E11.

$\square$ upilumab is a novel medication that is approved by the US Food and Drug Administration to treat moderate to severe atopic dermatitis (AD) in patients 6 years and older. Dupilumab is an injectable fully human monoclonal antibody. It provides a giant leap toward a better quality of life for patients with AD. Dupilumab works by binding to the shared $\alpha$ subunit of the IL-4 receptor (IL-4R), thus inhibiting IL-4 and IL-13 from using that signaling pathway. The documented sideeffect profile includes injection-site reaction, keratitis, nasopharyngitis, and headache. ${ }^{1}$

We initiated off-label treatment with dupilumab in 3 adult patients who had a history of long-standing adult-onset dermatitis confirmed by histopathology. The 3 patients received a loading dose of $600 \mathrm{mg}$ subcutaneously, followed by $300 \mathrm{mg}$ every other week. Following treatment, the patients had expansion of their disease, with features consistent with cutaneous T-cell lymphoma (CTCL) on subsequent biopsies. These 3 cases demonstrate the well-known adage that the diagnosis of CTCL often requires multiple biopsies performed over time. Although dupilumab has proved efficacious and safe for treating $\mathrm{AD}$, dermatologists should be cautious before starting this medication in an adult who has new-onset dermatitis and no history of atopy.

\section{Case Reports}

Patient 1-A 61-year-old man presented to dermatology after being lost to follow-up for several years and was started on dupilumab for long-standing nonspecific eczematous dermatitis based on histopathology. He had a pruritic rash of 10 years' duration that had been biopsied multiple times and was found to be consistent with dermatitis and lichen simplex chronicus (Figure 1). He had been treated with triamcinolone ointment $0.1 \%$ and

Drs. Hollins and Wirth are from the Department of Dermatology, Penn State Milton S. Hershey Medical Center. Dr. Fulchiero is from Keystone Dermatology \& Center for Skin Surgery, Altoona, Pennsylvania. Dr. Foulke is from the Department of Dermatology, University of North Carolina, Chapel Hill. The authors report no conflict of interest.

Correspondence: L. Claire Hollins, MD, Department of Dermatology, Penn State Milton S. Hershey Medical Center, 500 University Dr, Ste 4300, HU14, UPC III, Hershey, PA 17033 (Ihollins@pennstatehealth.psu.edu).

doi:10.12788/cutis.0074 
narrowband UVB as often as 3 times weekly over many years. The patient also had a history of idiopathic CD4 lymphopenia with consistently negative tests for human immunodeficiency virus.

At approximately the same time as dupilumab was initiated, he was started on $60 \mathrm{mg}$ daily of prednisone by his pulmonologist because of a history of restrictive lung disease of unknown cause. While taking prednisone, he experienced notable improvement in his skin condition; however, as he was slowly tapered off prednisone, he noted remarkable worsening of the dermatitis. Dupilumab was discontinued. Two more biopsies were performed; findings on both were consistent with mycosis fungoides (MF)(Figure 2).

Patient 2-A 52-year-old man presented with indurated, red, scaly plaques on the legs and arms. Initial biopsy was consistent with psoriasiform dermatitis that was thought to be due to a primarily eczematous process. Because of the clinical suspicion of psoriasis, the patient was at first treated with topical betamethasone and eventually was transitioned to multiple injectable biologics without improvement. There was no response to multiple psoriasis treatments, and the original pathology report was re-reviewed. The report noted a substantial eczematous component; therefore, a decision was made to transition him to dupilumab. He also was at first provided with a prednisone taper due to the severity of the cutaneous disease.

Initially, the patient noted $15 \%$ to $20 \%$ improvement; however, after 6 injections, dupilumab appeared to lose efficacy. Due to a lack of response to multiple biologic medications as well as dupilumab, another biopsy was performed. Findings were consistent with MF.


FIGURE 1. $A$ and $B$, Histopathologic findings from a biopsy of the hand just prior to dupilumab treatment (H\&E, original magnifications $\times 4$ and $\times 10$ ). Spongiosis and elongation of the rete ridges were seen. Parakeratosis and neutrophils were seen within the stratum corneum. The superficial dermis had a mixed inflammatory infiltrate.

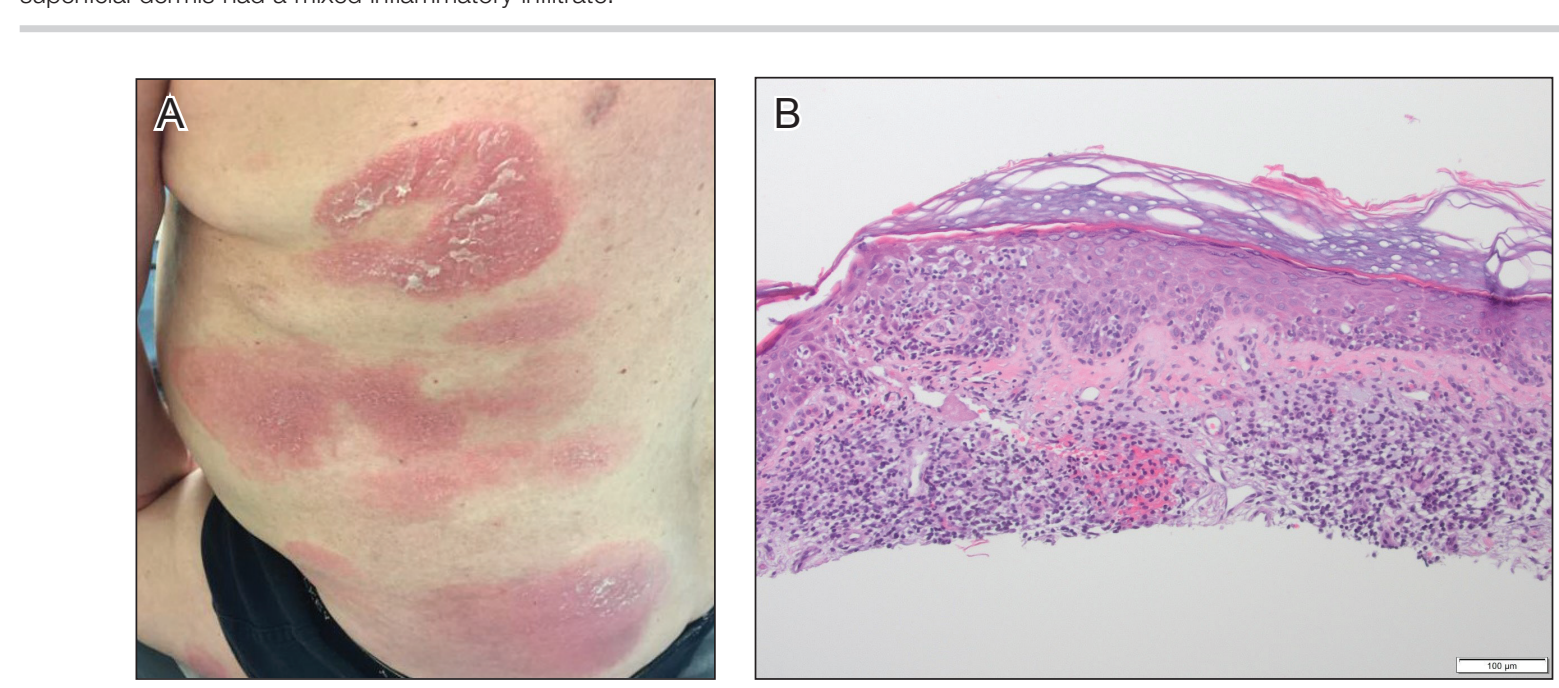

FIGURE 2. A, Red, annular to digitate plaques with overlying scale on the trunk after treatment with dupilumab. B, Psoriasiform hyperplasia of the epidermis with overlying crust and an increased number of lymphocytes extending into the epidermis were seen on biopsy of the back $(\mathrm{H} \& \mathrm{E}$, original magnification $\times 10)$. 
Patient 3-A 60-year-old woman with diffuse, pruritic, and erythematous dermatitis of 3 years' duration was referred from an outside dermatology group. Prior biopsies were consistent with eczematous dermatitis. However, because 1 isolated plaque demonstrated findings consistent with psoriasis, she was started on guselkumab, which was discontinued after 12 weeks of therapy for lack of efficacy. The patient also had been treated with a short course of narrowband UVB and topical corticosteroids without benefit.
Upon initial evaluation in our clinic, there was concern for Sézary syndrome; however, peripheral blood studies were normal, and there was no monoclonal spike or irregularity in the patient's Sézary flow cytometry panel. A biopsy demonstrated lichenoid dermatitis, possibly consistent with drug eruption. All supplements and likely medication culprits were discontinued without improvement.

Prior to follow-up in our clinic, the patient was again evaluated by an outside dermatologist and started on dupilumab. After 3 doses, she discontinued the

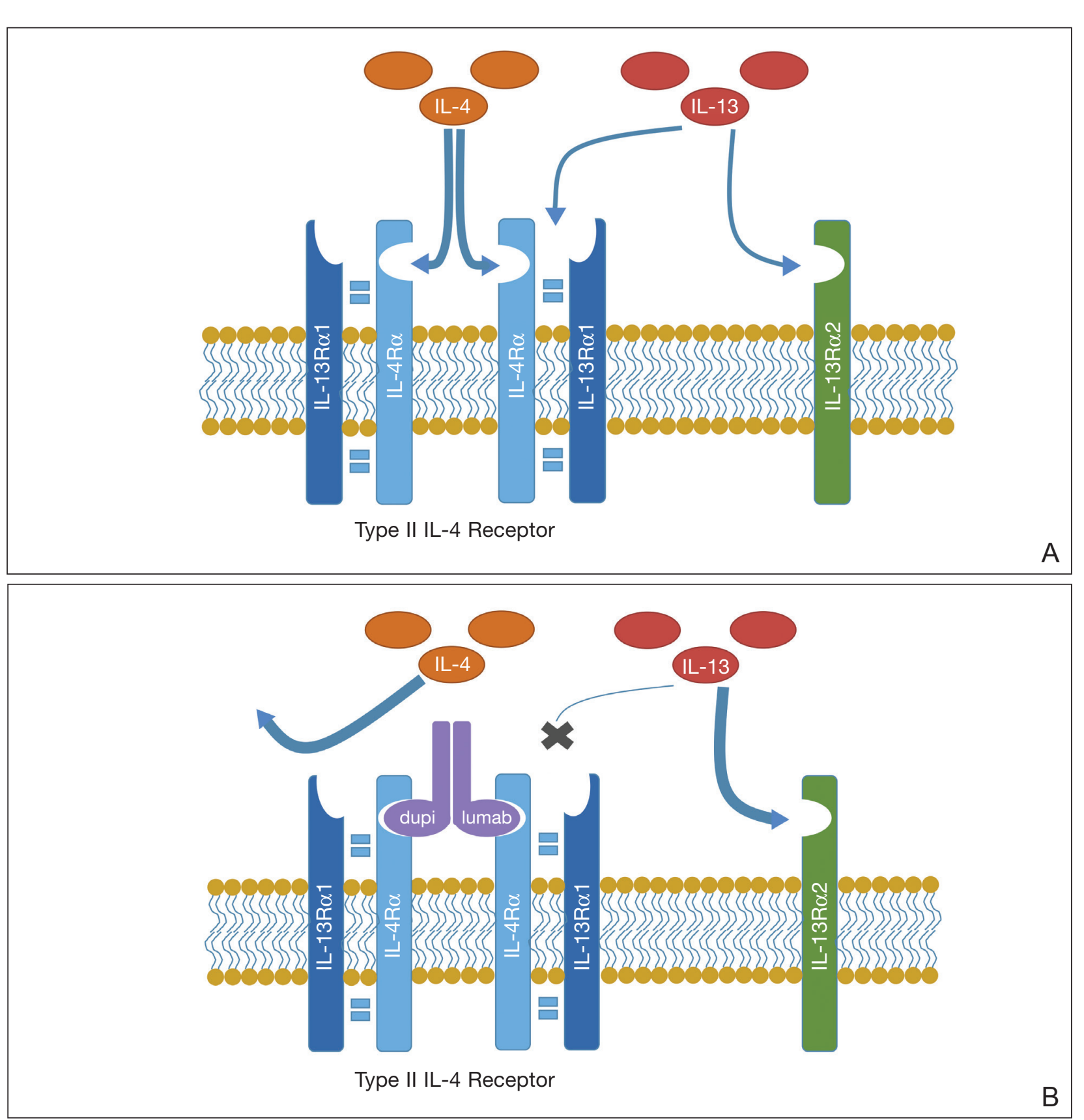

FIGURE 3. Proposed mechanism for promotion of cutaneous T-cell lymphoma (CTCL) by dupilumab. A, IL-4 and IL-13 bind to the type II IL-4 receptor (IL-4R). IL-13 also may bind at a distinct IL-13 receptor $\alpha 2$ (IL-13R $\alpha 2)$ site. B, Following blockade of the type II IL-4R, reduced binding at IL-4R may result in increased free IL-13 available for binding at the IL-13R 2 site, which may have a role in promoting CTCL proliferation. 
medication because there was no improvement in the cutaneous symptoms. Findings on repeat biopsy following dupilumab treatment were consistent with MF.

\section{Comment}

Mycosis fungoides is a rare chronic T-cell lymphoma that can smolder for decades as nonspecific dermatitis before declaring itself fully on skin biopsy. ${ }^{2}$ In many cases, MF masquerades as eczema, psoriasis, contact dermatitis, or other dermatitides, and it often responds to the same medications, making diagnosis even more challenging. Treatment options include topical steroids, narrowband UVB, topical nitrogen mustard, topical carmustine, and bexarotene gel for early-stage disease. ${ }^{3}$ Although it cannot be determined which patients will progress, some do, and therapies must then be upgraded.

We reported 3 patients with adult-onset dermatitis and multiple biopsies demonstrating nondiagnostic findings, which, in retrospect, likely represented early smoldering CTCL. Each of these patients was treated with dupilumab because multiple biopsies demonstrated findings consistent with nondiagnostic dermatitis, along with a lack of response to standard therapies. In all 3 cases, however, the patients had no history of eczema or atopy. After starting dupilumab, each patient had an acute exacerbation of dermatitis; immediately thereafter, biopsies were consistent with CTCL.

These patients most likely had smoldering CTCL that expressed itself fully after dupilumab was started. Biologic medications and their effects on the immune system have been shown to have multiple unanticipated effects on the skin. ${ }^{4-6}$ We are not insinuating that dupilumab was the cause of our patients having developed CTCL, but we do propose that the underlying interplay of dupilumab with the immune system might have accelerated progression of underlying CTCL, resulting in the lymphoma presenting itself clinically and histopathologically. We also must mention that all 3 cases could represent a"true, true, and unrelated" phenomenon.

A proposed mechanism for how dupilumab might hasten progression of CTCL is based on a functional increase of IL-13 available for binding at the IL-13 receptor (IL-13R) $\alpha 2$ site following blockade of the IL-13R $\alpha 1$ site by dupilumab (Figure 3). The pathway that is blocked by dupilumab provides improvement in $\mathrm{AD}$ by blocking the $\alpha$ subunit of the IL- $4 \mathrm{R}$, making it a receptor antagonist for both IL-4 and IL-13. The IL-4R forms a heterodimer with both $\gamma \mathrm{c}$ and separately with IL-13R $\alpha 1$. As a result, IL-4 and IL-13 cannot bind to their respective targets; thus, downstream signaling that is required for $\mathrm{AD}$ is halted. ${ }^{7} \mathrm{IL}-13$, in addition to IL-4R, also binds to an IL-13R $\alpha 2$. IL-13 and both of its receptors are upregulated in CTCL, particularly IL-13R $\alpha 2 .^{8}$

One of the principal ways that CTCL survives is through autocrine signaling, inducing more IL-13 and more IL-13R $\alpha 2$, which is not seen in normal skin. ${ }^{8}$ Autocrine signaling plays a critical role in cancer activation and in providing self-sustaining growth signals to tumors. ${ }^{9}$ In addition, it has been documented that IL-13R $\alpha 2$ has a higher affinity for IL-13 than the affinity of IL-13R $\alpha 1 .{ }^{10}$ As such, when the dupilumab receptor is blocked, our proposed mechanism of acceleration of CTCL is based on a functional increase in IL-13 available for binding at the IL-13R $\alpha 2$ site, following indirect blockade of the $\alpha 1$ receptor with dupilumab, which effectively increases available IL-13 to be shunted down the tumorigenic pathway.

We recognize that this proposed mechanism is a theory; additionally, it should be noted that dupilumab is approved only for the treatment of $\mathrm{AD}$ and asthma. In our 3 cases, we used dupilumab off label in patients who did not have a clear case of AD or a childhood history of the disease.

When screening patients for the use of dupilumab, it is important to treat only those who have a classic history of moderate to severe $\mathrm{AD}$, including itch, family history, and rash in the classic atopic distribution. We propose that these cases represent potential exacerbation of extant CTCL following exposure to dupilumab.

The manufacturer of dupilumab has reported 1 case of stage IV MF in a 57-year-old man 48 days after the first dose of dupilumab, leading to permanent discontinuation. The patient had ongoing disease at the time of the report, and the manufacturer stated that use of dupilumab was unrelated to disease. ${ }^{11}$ Studies are needed to explore any potential immunologic link between dupilumab and progression of CTCL.

\section{REFERENCES}

1. Raedler LA. Dupixent (dupilumab) first biologic drug approved for patients with moderate-to-severe atopic dermatitis. Am Health Drug Benefits. 2018;11:58-60.

2. Skov AG, Gniadecki R. Delay in the histopathologic diagnosis of mycosis fungoides. Acta Derm Venereol. 2015;95:472-475.

3. Ramsay DL, Meller JA, Zackheim HS. Topical treatment of early cutaneous T-cell lymphoma. Hematol Oncol Clin North Am. 1995; 9:1031-1056.

4. Mazloom SE, Yan D, Hu JZ, et al. TNF- $\alpha$ inhibitor-induced psoriasis: a decade of experience at the Cleveland Clinic [published online December 18, 2018]. J Am Acad Dermatol. doi: 10.1016/j.jaad.2018.12.018.

5. Tierney E, Kirthi S, Ramsay B, et al. Ustekinumab-induced subacute cutaneous lupus. JAAD Case Rep. 2019;5:271-273.

6. Orrell KA, Murphrey M, Kelm RC, et al. Inflammatory bowel disease events after exposure to interleukin 17 inhibitors secukinumab and ixekizumab: postmarketing analysis from the RADAR ("Research on Adverse Drug events And Reports") program. J Am Acad Dermatol. 2018;79:777-778.

7. Sastre J, Dávila I. Dupilumab: a new paradigm for the treatment of allergic diseases. J Investig Allergol Clin Immunol. 2018;28:139-150.

8. Geskin LJ, Viragova S, Stolz DB, et al. Interleukin-13 is over-expressed in cutaneous T-cell lymphoma cells and regulates their proliferation. Blood. 2015;125:2798-2805.

9. Barderas R, Bartolomé RA, Fernandez-Aceñero MJ, et al. High expression of IL-13 receptor $\alpha 2$ in colorectal cancer is associated with invasion, liver metastasis, and poor prognosis. Cancer Res. 2012;72:2780-2790.

10. Andrews A-L, Holloway JW, Puddicombe SM, et al. Kinetic analysis of the interleukin-13 receptor complex. J Biol Chem. 2002;277:46073-46078.

11. Data on file. Tarrytown, NY: Regeneron Pharmaceuticals, Inc; 2017. 